\title{
AVALIAÇÃO DO DESEMPENHO DE MÉTODOS DE ESTIMATIVA DE EVAPOTRANSPIRAÇÃO POTENCIAL PARA A REGIÃO NORTE DE RECIFE-PE
}

\author{
Alexsandro Oliveira da Silva ${ }^{1}$, Enio Farias de França e Silva ${ }^{2}$, Geber Barbosa de Albuquerque Moura $^{3}$, \\ Pabricio Marcos Oliveira Lopes ${ }^{4}$
}

\begin{abstract}
RESUMO
A evapotranspiração é um dos principais parâmetros para um adequado manejo da irrigação e, por isto, a escolha do modelo para sua estimativa deve ser rígida e criteriosa. Avaliou-se o desempenho de nove métodos de evapotranspiração de referência (ETp) na região norte da cidade de Recife/PE, através de dados coletados de uma estação meteorológica e tanque "Classe A" localizados na Universidade Federal Rural de Pernambuco entre os anos de 2009 e 2011. Os dados estimados foram correlacionados por meio de regressão linear tendo como padrão o método Penman-MonteithFAO 56. A avaliação de desempenho dos métodos foi baseada nos coeficientes de correlação "r" de concordância "d" de Willmott e o índice de desempenho "c". As melhores estimativas de ETp para as condições climáticas de Recife foram obtidas pelo método de Radiação solar, Camargo, Makkink e Priestley-Taylor, todos com índice de desempenho entre 0,80 e 0,98 (bom a ótimo). Os métodos de Hargreaves-Samani e Blaney-Criddle tiveram índices de desempenho entre bom e regular $(0,80$ a 0,60). Os métodos de ETp do tanque Classe A e Linacre obtiveram os piores índices de desempenho "c" (de 0,40 a 0,70$)$ dentre todas as avaliações realizadas.
\end{abstract}

Palavras-chave: Penman-Monteith-FAO 56, Blaney-Criddle e Priestley-Taylor.

\section{ABSTRACT \\ EVALUATION OF METHODS TO ESTIMATE POTENTIAL EVAPOTRANSPIRATION IN NORTHERN RECIFE-PE}

Evapotranspiration is one of the key factors determining efficiency of irrigation management. In the absence of climate data, the choice of a model for its estimation should be strictly criteria based. In this study the performance of nine methods of estimating potential evapotranspiration (ETp) in the northern Recife/PE were evaluated, using the data collected from a weather station and "Class A" pan located in the Federal Rural University of Pernambuco during the period of 2009 and 2011. Estimated data were correlated by linear regression, using FAO-Penman-Monteith method as standard. Performance of methods was based on correlation coefficients " $r$ ", agreement "d" of Willmott and the performance index "c". The best ETp estimates were obtained by the methods of solar radiation, Camargo, and Makkink and Priestley-Taylor, all with "c" index between 0.80 and 0.98 , considered good to best. The methods of HargreavesSamani and Blaney-Criddle had "c"index in the range of 0.6 to 0.89 , varying according to the mean of one, three, seven and ten days. The methods "Class A" pan, and Linacre had lowest "c" indexes varying between 0.40 and 0.70 .

Keywords: FAO-Penman-Monteith, Blaney-Criddle e Priestley-Taylor.

\section{Recebido para publicação em 14/03/2011. Aprovado em 08/09/2011.}

1- Engenheiro Agrônomo, Mestrando em Irrigação e Drenagem - Dep. de Engenharia Rural, UNESP, Botucatu -SP, e-mail: alexsandro_oliveira01@hotmail.com.

2- Engenheiro Agrícola, Professor Adjunto do Dep. de Tecnologia Rural, UFRPE, Recife-PE, e-mail: enio.silva@dtr.ufrpe.br.

3- Meteorologista, Professor Associado do Dep. de Agronomia, UFRPE, Recife-PE, email: geber@depa.ufrpe.br.

4- Meteorologista, Professor Adjunto do Dep. de Agronomia, UFRPE, Recife-PE, email: pabriciope@gmail.com. 


\section{INTRODUÇ̃̃O}

A evapotranspiração pode ser definida como um processo combinado de transferência de água do solo para atmosfera, incluindo a evapotranspiração direta da água do solo e o processo de transpiração através dos tecidos vegetais (Turco et al., 2005). Devido à grande necessidade de diminuir os gastos excessivos com água, as informações quantitativas da evapotranspiração são de grande importância na avaliação da severidade, distribuição e frequência dos déficits hídricos, elaboração de projetos de manejo de sistemas de irrigação e drenagem (HENRIQUE; DANTAS, 2007). Por estes e outros motivos diversas pesquisas com métodos indiretos de evapotranspiração vem sendo realizadas por todo o país comparando um método padrão, seja ele lisímetros de pesagem ou por métodos indiretos como Penman-Monteith FAO56, entre outros com os demais métodos, para a obtenção de um manejo adequado da irrigação. Assim, economiza-se dinheiro e recursos naturais como a água.

É preciso, portanto, buscar novas metodologias e práticas para estimar a evapotranspiração potencial com resultados satisfatórios, principalmente em regiões onde não existem dados climatológicos suficientes e confiáveis. De acordo com Hargreaves \& Felow (1994) a padronização das condições do local para a coleta de dados climáticos e confiabilidade dos mesmos são tão importantes quanto à escolha da equação usada para estimativa da ETp. Portanto, é necessário estudos para facilitar a vida do produtor rural no que diz respeito aos modelos matemáticos propostos e as suas correlações com o método padrão recomendadas pela $\mathrm{FAO}$, que é o método de Penman-Monteith.

Para a região metropolitana do Recife, em que a pratica da agricultura sustentável ainda é fonte de renda para famílias de baixa renda, a quantificação da evapotranspiração assume particular importância devido à demanda hídrica da região se apresentar variável ao longo do ano, pois o desperdício e a falta de água são fatores preponderantes no desenvolvimento das culturas e principalmente na produtividade, devido ao déficit hídrico causado. Segundo Penman (1963), as limitações dos métodos são reconhecidas tanto por seus críticos como por seus autores, mas enquanto prosseguem as buscas por melhores soluções, estes métodos podem fornecer valores de consumo de água para uso no balanço hídrico.

Devido aos problemas com dados climatológicos em diversas regiões pesquisadores como Silva et al. (2005) avaliaram a eficiência dos métodos de estimativa de Evapotranspiração de referencia em Petrolina- PE, usando o coeficiente de determinação média e a média do desvio padrão e concluíram que na ausência de dados de insolação e velocidade do vento, a evapotranspiração de referencia pelo método de FAO-Penman-Monteith pode ser substituída, com razoável precisão, pelo método de Hargreaves na escala diária. Procurando estimar a demanda hídrica para a cultura do citrus em AraraquaraSP, Vescove e Turco (2005) obtiveram resultados no método do Tanque "Classe A" satisfatórios quando obedecidas as equações de regressão determinadas. Oliveira et al. (2008), trabalhado no agreste pernambucano com lisímetro de pesagem, concluíram que a ETo estimada pela Radiação Solar foi a que mais se ajustou aos valores do lisímetro.

Diante do exposto, esse trabalho objetivou comparar diferentes métodos indiretos de estimativa de evapotranspiração potencial para a Região Norte de Recife, PE, considerando o método da Penman-Monteith-FAO 56, como padrão.

\section{MATERIAL E MÉTODOS}

A pesquisa foi desenvolvida na região norte de Recife no Departamento de Tecnologia Rural (DTR) da Universidade Federal Rural de Pernambuco ( $-8^{\circ} 01^{\prime} 05^{\prime}$, longitude: $-34^{\circ} 56^{\prime} 48^{\prime \prime}$ e altitude: $6,48 \mathrm{~m}$ ). O clima, segundo Köppen é classificado como tropical chuvoso (tipo As' a Ams'). A região apresenta temperatura média anual de $27^{\circ} \mathrm{C}$ e precipitação anual acima de $1700 \mathrm{~mm}$. O local onde estão instaladas a estação meteorológica automática e o tanque classe " $\mathrm{A}$ " possui uma área de $12 \times 12 \mathrm{~m}$ coberta com grama

\section{REVENG}

$$
\text { 163-174 p. }
$$

ENGENHARIA NA AGRICULTURA, VIÇOSA - MG, V.20 N.2, MARÇO / ABRIL 2012 
batatais na altura de $10 \mathrm{~cm}$. Nesse local foram coletados valores diários de temperaturas do ar máximas e mínimas, umidade relativa, radiação solar, precipitação e velocidade do vento a 2 metros de altura além de dados de evaporação do tanque nos anos de 2009 a 2010.

Com o propósito de tornar os dados agrometeorológicos mais homogêneos, foram eliminadas informações discrepantes, incompletas ou inconsistentes. A ETp foi estimada por meio de nove métodos indiretos os quais são: PenmanMonteith-FAO 56 (ETpPM), Radiação solar (ETpRS), Blaney-Criddle (ETpBC), PriestleyTaylor (ETpPT), Linacre (ETpL), Makkink (ETpMK), Tanque "Classe A" (ETpCL), Camargo (ETpCM), Hargreaves-Samani (ETpHS). As equações dos métodos estão detalhadamente descritas em Pereira, et al. (1997).

Os métodos indiretos foram estimados na escala de um, três, sete e dez dias. Utilizou-se a regressão linear simples, correlação (r), índice de concordância (d), índice de desempenho (c) e erro padrão da estimativa (EPE) para avaliação de desempenhos dos métodos, tomando como método padrão o FAO-Penman-Monteith. A precisão foi dada pelo coeficiente de determinação, a qual indica o grau em que a regressão explica a soma do quadrado total. Conforme Willmott (1982), o índice "d" é dado pelas seguintes equações:

$$
d=1-\frac{\sum_{i=1}^{n}\left(\hat{y}_{i}-y_{i}\right)^{2}}{\sum_{i=1}^{n}\left(\left|\hat{y}_{i}\right|+\left|y_{i}\right|\right)^{2}}
$$

$\hat{y}_{i}=\hat{y}_{i}-\bar{y}$

$y_{i}=y_{i}-\bar{y}$

em que

d = índice de concordância adimensional variando de 0 a 1 ;

$\hat{y}_{i}=$ ETp estimada pelo método indireto $\left(\mathrm{mm} \mathrm{dia}^{-1}\right)$;

$y=$ ETp medida pelo método padrão $\left(\mathrm{mm} \mathrm{dia}^{-1}\right)$;

$\bar{y}=$ média dos valores do método padrão $\left(\mathrm{mm} \mathrm{dia}^{-1}\right)$.
O coeficiente de correlação (r) foi definido por meio da seguinte equação:

$$
r=\frac{\sum_{i=1}^{n} X_{i} Y_{i}-\frac{\left(\sum_{i=1}^{n} X_{i}\right)\left(\sum_{i=1}^{n} Y_{i}\right)}{N}}{\sqrt{\left[\sum_{i=1}^{n} X_{i}^{2}-\frac{\left[\sum_{i=1}^{n} X_{i}\right]^{2}}{n}\right]\left[\sum_{i=1}^{n} Y_{i}^{2}-\frac{\left.\left[\sum_{i=1}^{n} Y_{i}\right]^{2}\right]}{n}\right]}}
$$

em que

$\mathrm{r}=$ coeficiente de correlação;

$\mathrm{X}_{\mathrm{i}}=\mathrm{ETp}$ estimada pelo método padrão $\left(\mathrm{mm} \mathrm{dia}^{-1}\right)$; e $\mathrm{Y}_{\mathrm{i}}=$ ETp estimada pelos métodos indiretos $\left(\mathrm{mm} \mathrm{dia}^{-1}\right)$.

Para a avaliação do desempenho dos métodos foi utilizado o índice (c) proposto por Camargo e Sentelhas (1997) para indicar o desempenho dos métodos que reúne os coeficientes de correlação (r) e o de concordância (d) conforme equação (5) e Quadro 1:

$$
c=r \cdot d
$$

em que

$\mathrm{c}=$ índice de "c" de desempenho;

$\mathrm{d}$ =índice de concordância, adimensional, variando de 0 a 1 ;

$\mathrm{r}=$ coeficiente de determinação.

Quadro 1. Critério de interpretação do desempenho dos métodos de estimativa da ETp pelo índice "c" de Camargo e Sentelhas (1997)

\begin{tabular}{cc}
\hline Valor de "c" & Desempenho \\
\hline$>0,85$ & Ótimo \\
0,76 a 0,85 & Muito Bom \\
0,66 a 0,75 & Bom \\
0,61 a 0,65 & Mediano \\
0,51 a 0,60 & Sofrível \\
0,41 a 0,50 & Mau \\
$\leq 0,40$ & Péssimo \\
\hline
\end{tabular}


O erro padrão de estimativa (EPE) mede a dispersão dos valores amostrados ao redor da reta de regressão, definido pela expressão:

$E P E=\sqrt{\frac{\sum_{i=1}^{n}\left(y_{i}-\hat{y}_{i}\right)^{2}}{n-1}}$

em que

$\mathrm{EPE}=$ erro padrão de estimativa;

$\hat{y}_{i}=$ ETp estimada pelo método padrão;

$y_{i}=$ ETp estimada pelos demais métodos;

$\mathrm{n}=$ número de observações.

\section{RESULTADOS E DISCUSSÃO}

Na Figura 1, (a, b e c) observa-se a variação anual da temperatura do ar e umidade relativa do ar e velocidade do vento, obtida na estação meteorológica de Recife. Os valores médios da irradiância solar, determinados para a latitude de Recife ( $\left.-8^{\circ} 01^{\prime} 05^{\prime \prime}\right)$, encontram-se na Figura 1d. A temperatura média mensal do ar variou entre $25 \mathrm{a} 29^{\circ} \mathrm{C}$, respectivamente, as menores temperaturas foram encontradas para omês de julho e as maiores em março. Os menores valores de umidade relativa encontraram-se em março e os maiores em junho com 72 e $80 \%$, respectivamente. A velocidade do vento oscilou consideravelmente apenas em agosto e setembro, com medias acima de $4,5 \mathrm{~m} \mathrm{~s}^{-1}$. A radiação solar apresentou a menor média no mês de julho $\left(13,4 \mathrm{MJ} \mathrm{m}^{-2}\right.$ dia $\left.^{-1}\right)$ e a maior média em março $\left(23,6 \mathrm{MJ} \mathrm{m}^{-2} \mathrm{dia}^{-1}\right)$.

No Quadro 2 apresentam-se as médias mensais da ETp determinadas por meio dos métodos PenmanMonteith-FAO (ETpPM), Radiação solar (ETpRS), Blaney-Criddle (ETpBC), Linacre (ETpL), PriestleyTaylor (ETpPT), Makkink (ETpMK), Tanque "Classe A" (ETpCLA), Camargo (ETpCM), HargreavesSamani (ETpHS). Nota-se que os métodos
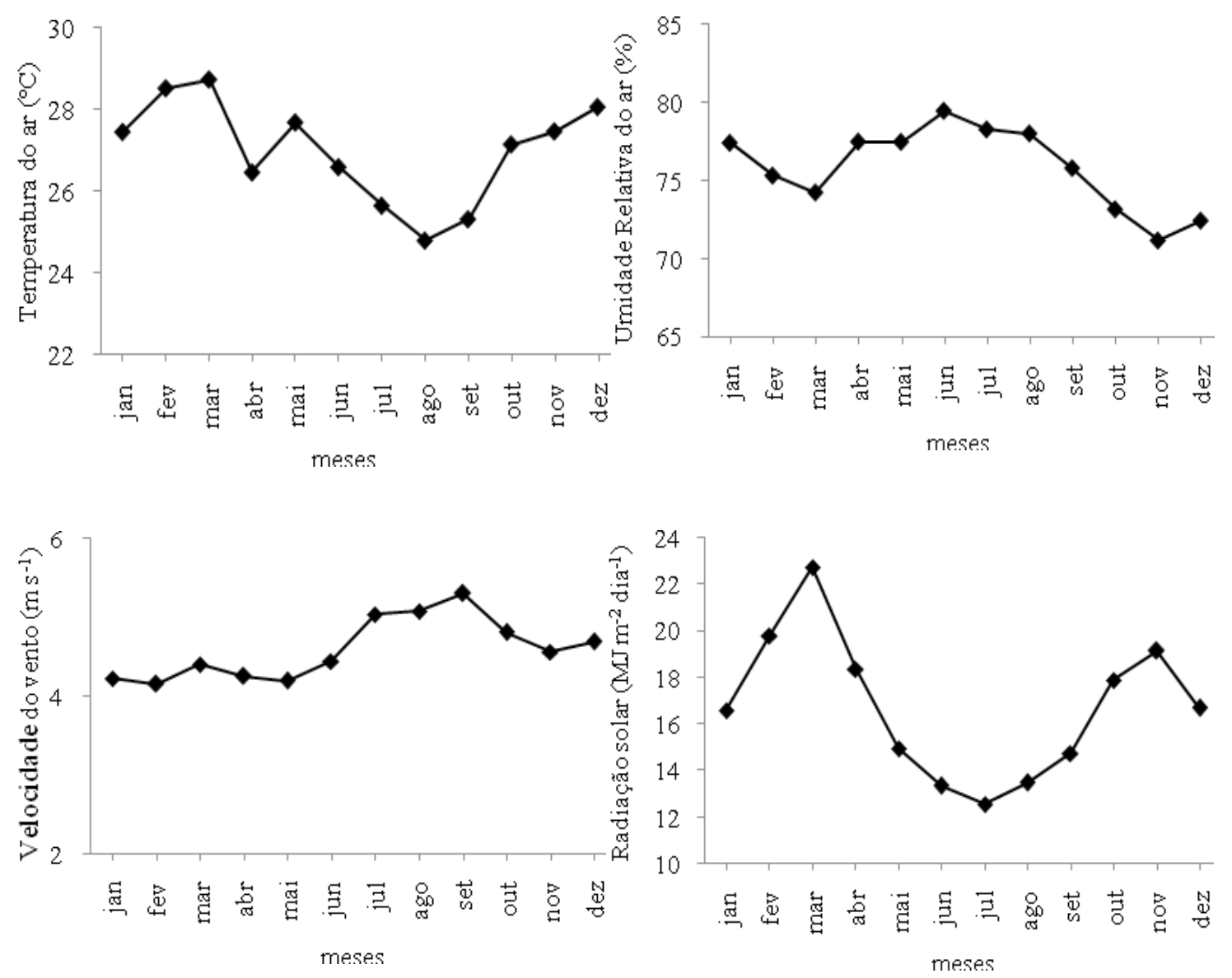

Figura 1. (a): Valores médios mensais da temperatura do ar; (b): Valores médios mensais da umidade relativa do ar; (c): Valores médios de velocidade do vento $\left(\mathrm{m} \mathrm{s}^{-1}\right)$; (d): Valores médios mensais da radiação solar. 
ETpRS, ETpMK, ETpL e ETpPT apresentaram tendências de superestimar a ETp em 66, 67, 47, $74 \%$, respectivamente. Os métodos ETpL e ETpPT apresentaram valores de ETp superiores ao método de ETpPM em todos os meses do ano. Já os métodos ETpCLA e ETpHS, em média, subestimaram ETp em 10 e $2,0 \%$.

No Quadro 3 mostra que o método de ETpCM e ETpRS apresentaram os melhores índices de desempenhos ( $\mathrm{c} \geq 0,90$ ), classificando-se como ótimo. Tagliaferre et al. (2010) obtiveram resultados semelhantes para a região de Eunápolis-BA e concluíram que o método de radiação pode ser utilizado.

Os métodos de ETpL e ETpCLA apresentaram os piores índices de desempenho $(0,45$ e 0,43$)$ quando comparados ao método de ETpPM. Estes resultados foram contraditórios aos apresentados por Mendonça et al. (2003) que, estimando diversos métodos de evapotranspiração para a região Norte - Fluminense-RJ, obtiveram índices superiores, superestimando o Método de Penman-Monteith -FAO 56 em escala diária.

Os métodos de ETpPT e ETpHS apresentaram os maiores índices de desempenho para valores estimados em escala de três dias. Estes resultados estão de encontro aos de Almeida et al. (2010), que obtiveram, em estudo de estimativa de ETp , os piores índices para ETpHS durante o ano, em quanto Cavalcante Junior et al. (2010), estimando a ETp para Mossoró-RN, apresentaram os maiores índices de desempenho para o método de ETpHS.

$\mathrm{Na}$ escala de três dias, os maiores EPE foram obtidos para os métodos de ETpMK $\left(0,67 \mathrm{mmd}^{-1}\right)$ e ETpCLA $\left(0,79 \mathrm{~mm} \mathrm{~d}^{-1}\right)$. Já os métodos de ETpCM e ETpHS apresentaram os maiores índices de desempenho (0,94 e 0,96 respectivamente), tido como ótimo seguido pelo método ETpRS $(0,87)$ para a escala de sete dias. Syperreck et al. (2008), em estudos com ETp no Estado do Paraná, mesmo em climas mais amenos, apresentaram resultados semelhantes para a ETpCM para a região estudada. Os métodos de Blaney-Criddle e Tanque "Classe A", apresentaram os piores índices de desempenhos com 0,60 e 0,53, respectivamente, e com EPE de 0,47 e $0,69 \mathrm{~mm} \mathrm{dia}^{-1}$. Os métodos de ETpL e ETpCLA apresentaram os piores índices de desempenho "c" com 0,44 e 0,43 e EPE de 0,43 e $1,18 \mathrm{~mm} \mathrm{dia}^{-1}$, subestimando os valores do método padrão. Vescove e Turco (2005) obtiveram resultados com o método do tanque "Classe A" superestimado os valores de ETpPM, evidenciando diferenças significativas entre os métodos.

Quadro 2. Variação dos valores médios mensais da ETp determinada pelo método padrão FAO-PenmanMonteith e pelos demais métodos* comparados, em mm dia ${ }^{-1}$

\begin{tabular}{lccccccccc}
\hline \multirow{1}{*}{ Mês } & \multicolumn{10}{c}{ Métodos (ETp) } \\
\cline { 2 - 10 } & PM & RS & BC & MK & L & PT & CLA & CM & HS \\
\hline Janeiro & 3,26 & 6,21 & 4,65 & 4,31 & 4,58 & 7,02 & 3,40 & 5,21 & 3,67 \\
Fevereiro & 3,38 & 6,10 & 4,83 & 3,45 & 6,15 & 7,32 & 4,22 & 6,33 & 4,47 \\
Março & 4,78 & 4,77 & 4,33 & 6,06 & 5,70 & 6,40 & 3,39 & 4,87 & 4,15 \\
Abril & 4,25 & 4,00 & 4,12 & 5,60 & 5,34 & 5,51 & 2,57 & 4,01 & 3,78 \\
Maio & 3,10 & 3,75 & 3,81 & 5,48 & 4,96 & 5,07 & 3,09 & 3,60 & 3,38 \\
Junho & 2,70 & 4,25 & 3,76 & 5,84 & 4,85 & 5,04 & 2,90 & 3,47 & 3,39 \\
Julho & 2,50 & 6,35 & 4,07 & 7,25 & 5,24 & 4,89 & 2,64 & 3,18 & 2,57 \\
Agosto & 2,65 & 6,88 & 5,11 & 7,38 & 5,54 & 5,95 & 2,65 & 3,89 & 2,59 \\
Setembro & 5,00 & 6,66 & 5,10 & 6,87 & 5,88 & 6,98 & 2,75 & 5,12 & 2,86 \\
Outubro & 3,25 & 5,66 & 5,45 & 3,88 & 5,82 & 6,54 & 3,36 & 4,87 & 3,79 \\
Novembro & 3,65 & 4,55 & 5,19 & 3,50 & 3,72 & 6,80 & 3,24 & 5,03 & 3,70 \\
Dezembro & 3,45 & 5,00 & 6,00 & 5,40 & 5,55 & 6,64 & 3,75 & 4,66 & 3,68 \\
\hline ETp média & 3,55 & 5,35 & 4,70 & 5,41 & 5,23 & 6,18 & 3,16 & 4,52 & 3,50 \\
\hline Dif. Relativa & $\%$ & 66,0 & 25,0 & 67,0 & 47,0 & 74,0 & $-10,0$ & 27,0 & $-2,0$ \\
\hline
\end{tabular}

*PM: Penman Monteith-FAO; RS:Radiação Solar; BC:Blaney-Criddle; MK: Makkink; L:Linacre; PT: PriestleyTaylor; CLA: Tanque classe "A”; CM: Camargo; HS: Hargreaves-samani. 
Quadro 3. Resumo estatístico referente às equações de regressão linear e ao desempenho dos métodos de cálculos da ETo nas escalas de um, três, sete e dez dias

\begin{tabular}{|c|c|c|c|c|c|c|}
\hline \multicolumn{7}{|c|}{ Escala diária } \\
\hline Métodos & $\mathrm{R}$ & EPE & $\mathrm{R}^{2}$ & $\mathrm{~d}$ & $\mathrm{c}$ & Desempenho \\
\hline Radiação Solar & 0,95 & 0,53 & 0,90 & 0,99 & 0,94 & Ótimo \\
\hline Blaney-Criddle & 0,82 & 0,50 & 0,67 & 0,94 & 0,77 & Muito bom \\
\hline Makkink & 0,98 & 0,89 & 0,99 & 0,88 & 0,88 & Ótimo \\
\hline Linacre & 0,52 & 0,54 & 0,26 & 0,88 & 0,45 & Mau \\
\hline Priestley-Taylor & 0,98 & 0,08 & 0,99 & 0,90 & 0,89 & Ótimo \\
\hline Tanque Classe "A" & 0,43 & 0,83 & 0,24 & 0,99 & 0,43 & Mau \\
\hline Camargo & 0,98 & 0,11 & 0,99 & 0,97 & 0,96 & Ótimo \\
\hline Hargreaves-Samani & 0,83 & 0,51 & 0,70 & 0,99 & 0,83 & Muito Bom \\
\hline \multicolumn{7}{|c|}{ Três dias } \\
\hline Métodos & $\mathrm{R}$ & EPE & $\mathrm{R}^{2}$ & $\mathrm{~d}$ & c & Desempenho \\
\hline Radiação Solar & 0,88 & 0,53 & 0,88 & 0,93 & 0,87 & Ótimo \\
\hline Blaney-Criddle & 0,56 & 0,49 & 0,31 & 0,98 & 0,55 & Sofrível \\
\hline Makkink & 0,87 & 0,67 & 0,75 & 0,88 & 0,76 & Muito Bom \\
\hline Linacre & 0,71 & 0,36 & 0,50 & 0,96 & 0,68 & Bom \\
\hline Priestley-Taylor & 0,98 & 0,10 & 0,99 & 0,89 & 0,89 & Ótimo \\
\hline Tanque Classe "A" & 0,48 & 0,79 & 0,22 & 0,99 & 0,47 & Mau \\
\hline Camargo & 0,99 & 0,13 & 0,98 & 0,97 & 0,96 & Ótimo \\
\hline Hargreaves-Samani & 0,90 & 0,32 & 0,82 & 0,99 & 0,89 & Ótimo \\
\hline \multicolumn{7}{|c|}{ Sete dias } \\
\hline Métodos & $\mathrm{R}$ & EPE & $\mathrm{R}^{2}$ & $\mathrm{~d}$ & c & Desempenho \\
\hline Radiação Solar & 0,93 & 0,48 & 0,86 & 0,93 & 0,87 & Ótimo \\
\hline Blaney-Criddle & 0,61 & 0,47 & 0,37 & 0,98 & 0,60 & Sofrível \\
\hline Makkink & 0,91 & 0,50 & 0,83 & 0,87 & 0,80 & Muito Bom \\
\hline Linacre & 0,77 & 0,29 & 0,60 & 0,96 & 0,74 & Bom \\
\hline Priestley-Taylor & 0,98 & 0,06 & 0,99 & 0,89 & 0,88 & Ótimo \\
\hline Tanque Classe "A" & 0,53 & 0,69 & 0,28 & 0,99 & 0,53 & Sofrível \\
\hline Camargo & 0,98 & 0,06 & 0,99 & 0,97 & 0,96 & Ótimo \\
\hline Hargreaves-Samani & 0,95 & 0,20 & 0,90 & 0,99 & 0,94 & Ótimo \\
\hline \multicolumn{7}{|c|}{ Dez dias } \\
\hline Métodos & $\mathrm{R}$ & EPE & $\mathrm{R}^{2}$ & $\mathrm{~d}$ & $\mathrm{c}$ & Desempenho \\
\hline Radiação Solar & 0,98 & 0,51 & 0,87 & 0,99 & 0,98 & Ótimo \\
\hline Blaney-Criddle & 0,90 & 0,45 & 0,82 & 0,94 & 0,85 & Muito Bom \\
\hline Makkink & 0,97 & 0,24 & 0,95 & 0,87 & 0,88 & Ótimo \\
\hline Linacre & 0,51 & 0,43 & 0,35 & 0,87 & 0,44 & Mau \\
\hline Priestley-Taylor & 0,98 & 0,06 & 0,99 & 0,89 & 0,88 & Ótimo \\
\hline Tanque Classe "A" & 0,43 & 1,18 & 0,24 & 0,99 & 0,43 & Mau \\
\hline Camargo & 0,98 & 0,11 & 0,99 & 0,97 & 0,96 & Ótimo \\
\hline Hargreaves-Samani & 0,52 & 0,53 & 0,64 & 0,90 & 0,75 & Bom \\
\hline
\end{tabular}

Analisando as Figuras 2, 3,4 e 5 verifica-se que os métodos de ETpRS, ETpMK, ETpPT e ETpCM, apesar de seus valores superestimarem a ETpPM e apresentam coeficiente de determinação a partir de 0,90 na maioria das escalas diárias. Os métodos de ETpBC e ETpCLA e ETpL apresentaram dados bastante dispersos nas diversas escalas temporais. $\mathrm{O}$ método de ETpHS apresentou um coeficiente de determinação considerado eficiente para sua utilização devido a sua praticidade e por exigir poucos dados climáticos da Região, como afirmado por Mendonça et al.(2003). Estes autores obtiveram resultados satisfatórios para ETpHS. Devido a utilização de maiores variáveis climáticas, como o método padrão, a ETpCM, ETpPT, ETpMK e ETpRS puderam obter resultados satisfatórios, sendo ambas 
utilizarem coincidentemente como variável a radiação solar. $\mathrm{O}$ método de $\mathrm{ETpBC}$ para a estimativa de evapotranspiração potencial, em médias de três dias, aumentou o índice de correlação referente à escala diária, enquanto a ETpL e ETpCLA mantiveram-se com valores que subestimam o método padrão, devi do a alta dispersão de dados.
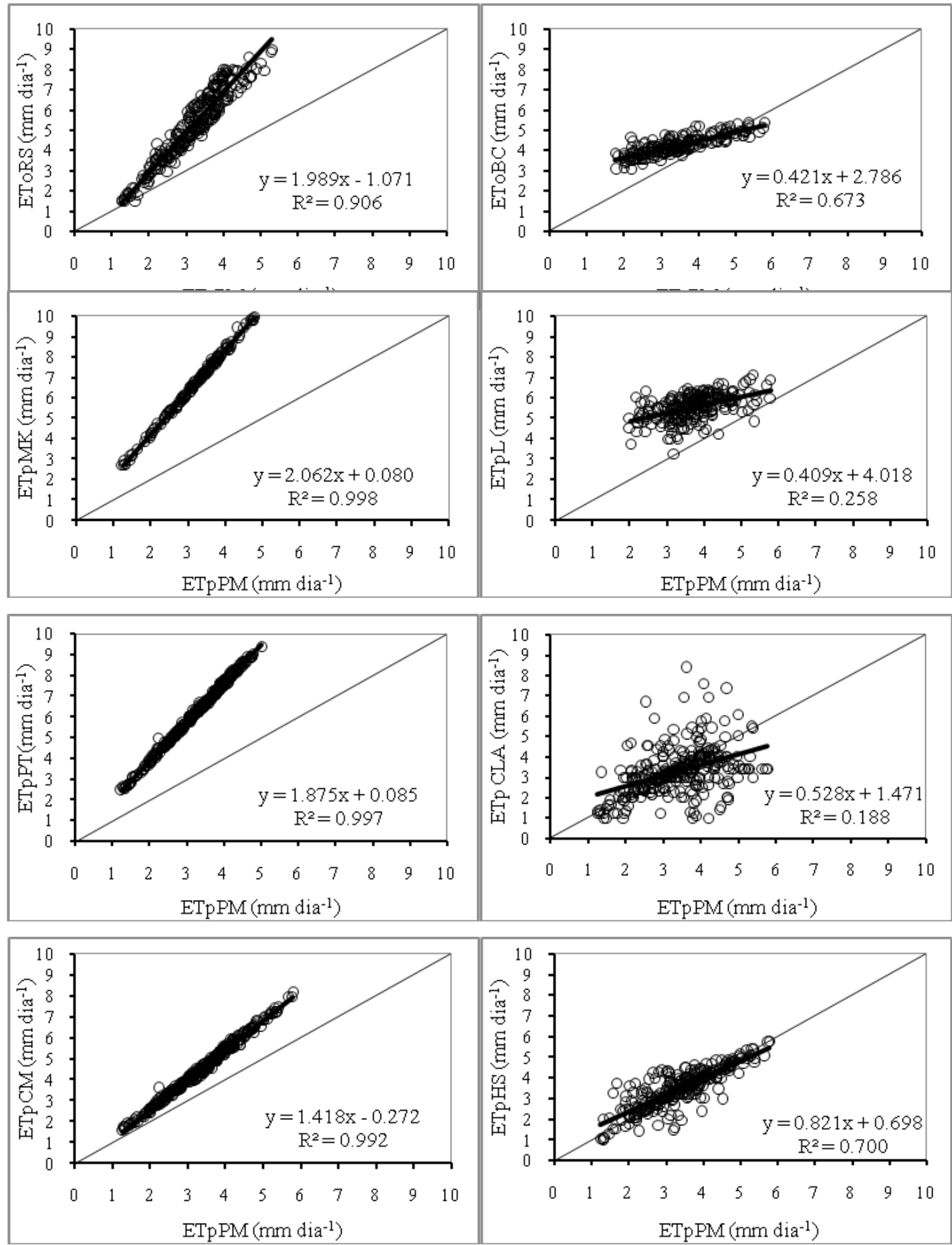

Figura 2. Correlação linear entre a ETp por Penman-Monteith-FAO 56 e estimada pelos demais métodos indiretos na escala diária. 

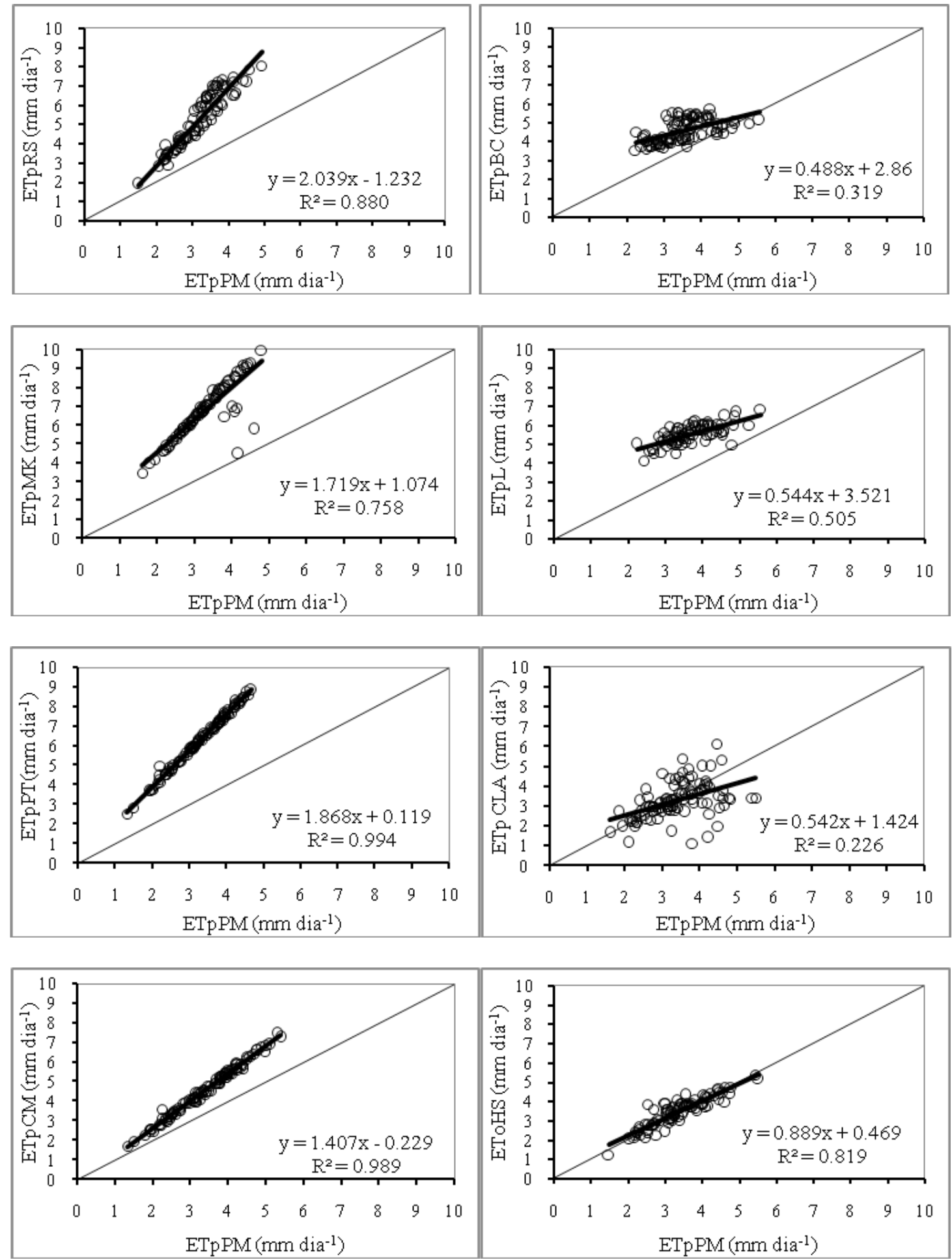

Figura 3. Correlação linear entre a ETp por Penman-Monteith-FAO 56 e estimada. 

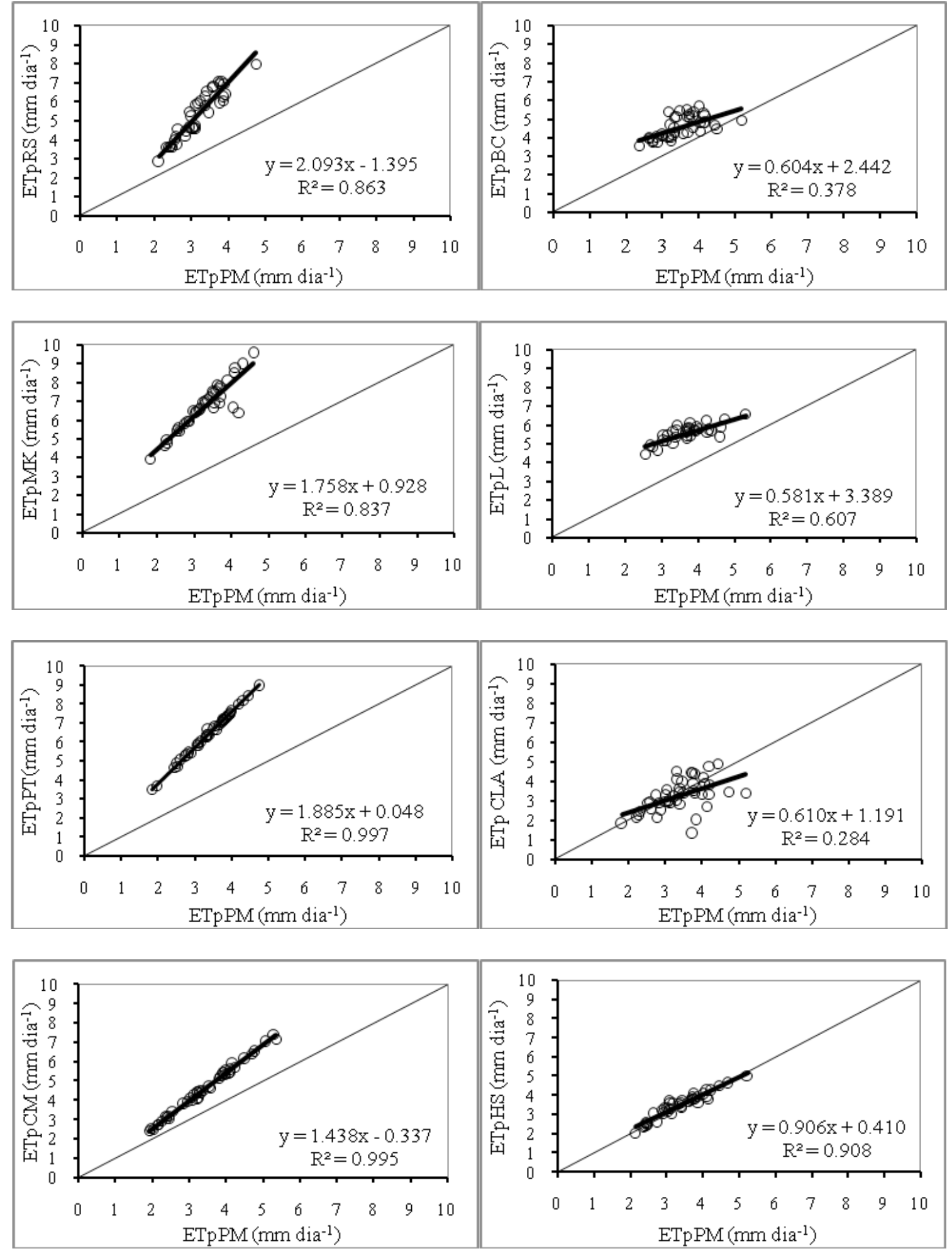

Figura 4. Correlação linear entre a ETp por Penman-Monteith-FAO 56 e estimada pelos demais métodos indiretos em média de sete dias. 

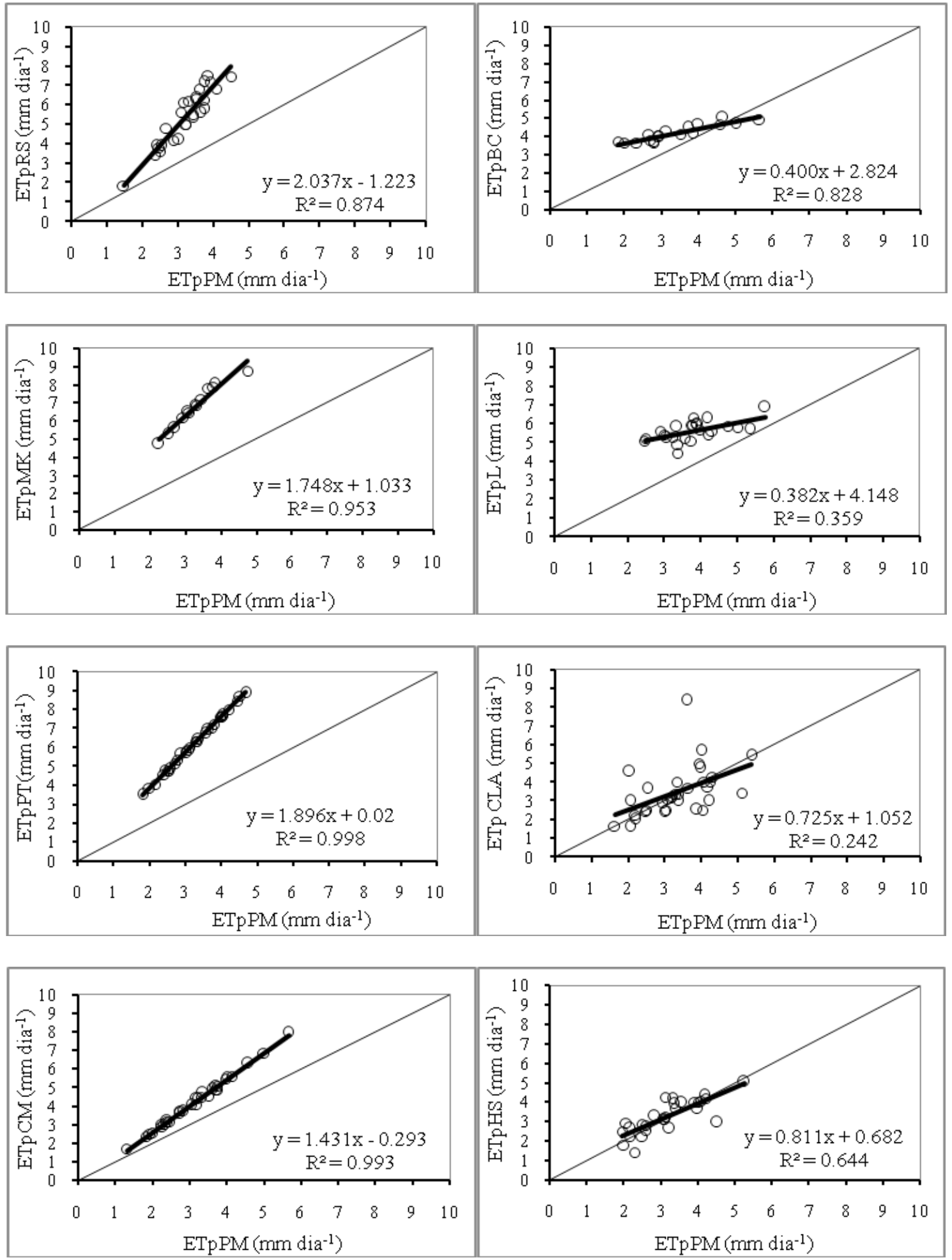

Figura 5. Correlação linear entre a ETp por Penman-Monteith-FAO 56 e estimada pelos demais métodos indiretos em média de dez dias.

\section{REVENG}

163-174 p. ENGENHARIA NA AGRICULTURA, VIÇOSA - MG, V.20 N.2, MARÇO / ABRIL 2012 


\section{CONCLUSÕES}

- Os métodos da radiação solar Camargo, Priestley-Taylor e Makkink apresentaram melhor desempenho para a estimativa da ETp em todos os períodos estudados;

- Os métodos do tanque "classe A" e Linacre, não apresentaram valores satisfatórios em nenhum dos períodos estudados, subestimando o método padrão FAO-Penman-Monteith; e

- Os métodos de Hargreaves-Samani e BlaneyCriddle foram variáveis nos períodos de tempo estudados apresentando valores satisfatórios apenas na escala de um, três e dez dias.

\section{REFERÊNCIAS BIBLIOGRÁFICAS}

ALMEIDA, B. M.; ARAUJO, E. M.; CAVALCANTE JUNIOR, E. G.; OLIVEIRA, J. B.; ARAUJO, E. M.; NOGUEIRA, B. R. C. Comparação de Métodos de Estimativa da ETo na escala mensal em Fortaleza-CE. Revista Brasileira de Agricultura Irrigada, Fortaleza, v.4, n.2, p.9398, abr/jun. 2010.

CAVALCANTE JUNIOR, E. G.; ALMEIDA, B. M.; OLIVEIRA, A. D.; SOBRINHO, J. E.; ARAUJO, E. M.; VIEIRA, R. Y. M. Estimativa de evapotranspiração de referência para a cidade de Mossoró-RN. Revista Brasileira de Agricultura irrigada, Fortaleza, v.4, n.2, p.87-92, abr/jun. 2010 .

CAMARGO, A. P.; SENTELHAS, P. C. Avaliação do desempenho de diferentes métodos de estimativa da evapotranspiração potencial no estado de São Paulo, Brasil. Revista Brasileira de Agrometeorologia, Santa Maria, v.5, n.1, p.89-97, jan/mar, 1997.

HARGREAVES, G. H.; FELlOW, A. S. C. E. Defining and using reference evapotranspiration. Journal of Irrigation and Drainage Egineering, New York, v.120, n.6. p.1132-1133, nov/dec. 1994.

HENRIQUE, F. A. N.; DANTAS, R. T. Estimativa da evapotranspiração de referência em Campina Grande, Paraíba. Revista Brasileira de Engenharia Agrícola e Ambiental, Campina Grande, v.11, n.6, p.594-599, nov/dez. 2007.

MENDONÇA, J. C.; SOUSA, E. F.; BERNARDO, S.; DIAS, G. P.; GRIPPA, S. Comparação entre métodos de estimativa da evapotranspiração de referência (ETo) na região Norte Fluminense, RJ. Revista Brasileira de Engenharia Agrícola e ambiental, Campina Grande, v.7, n.2, p.275-279, mai/ago. 2003.

OLIVEIRA, L. M. M.; MONTENEGRO, S. G. L.; AZEVEDO, J. R. G.; SANTOS, F. X. Evapotranspiração de Referencia na Bacia experimental do riacho da Gameleira PE, utilizando-se lisímetros e métodos indiretos. Revista Brasileira de Ciências Agrárias, Recife, v.3, n.1 p.58-67, jan/mar, 2008.

PEREIRA, V. N.; VILLA NOVA, N. A.; SEDIYAMA, G. C. Evapotranspiração. $1^{\text {a }}$ ed. Piracicaba: FEALQ, 1997. 183p.

PENMAN, H. L. Evaporation, transpiration and evapotranspiration. In: Vegetation and Hidrology. Farnham Royal: commonwealth Agricultural Bureaux, 1963, p.30-50.

SILVA, V. P. R.; FILHO, A.F.B.; SILVA, B.B.;CAMPOS, J.H. Avaliação de métodos de estimativa da evapotranspiração de referência. In: Congresso Brasileiro de Agrometeorologia, 14, 2005, Campinas. Anais... Campinas: Sociedade Brasileira de Agrometeorologia, 2005. CD Rom.

SYPERRECK, V. L. G.; KLOSOWSKI, E. S.; GRECO, M.; FURLANETTO, C. Avaliação de desempenho de métodos para estimativas de evapotranspiração de referência para a região de Palotina, Estado do Paraná. Acta scientiarum Agronomy, Maringá, v.30, n.5, p.603-609, set/ fev. 2008.

TURCO, J. E. P.; FARIA, M. T.; FERNANDES, E. J. Influência da forma de obtenção do saldo de radiação na comparação de métodos de estimativa 
da evapotranspiração de referência. Revista irriga, Botucatu, v.10, n.3, p. 215-228, jul/set. 2005.

TAGLIAFERRE, C.; SILVA, R. A. J.; ROCHA, F. A.; SANTOS, L. C.; SILVA, C. S. Estudo comparativo entre metodologias para determinação da evapotranspiração de referência em Eunápolis-BA. Revista Caatinga, Mossoró, v.23, n.1, p.103-111, jan/mar. 2010.
VESCOVE, H. V.; TURCO, J.E. P. Comparação de três métodos de estimativa da evapotranspiração de referência para a Região de Araraquara-SP. Revista Engenharia Agrícola, Jaboticabal, v.25, n.3, p.713-721, jul/set. 2005.

WILLMOTT, C. J. Some comments on the evaluation of model performance. Buletin of the American Meteorological Society, Lancaste, v.63, n.11, p.1309-1313, nov. 1982. 